\title{
Labyrinthe
}

$24 \mid 2006$ (2)

Faut-il être postcolonial?

\section{Alter, inter : académisme et postcolonial studies}

\section{Laurent Dubreuil}

\section{OpenEdition}

Journals

Édition électronique

URL : http://journals.openedition.org/labyrinthe/1248

DOI : $10.4000 /$ labyrinthe. 1248

ISSN : 1950-6031

Éditeur

Hermann

\section{Édition imprimée}

Date de publication : 15 juin 2006

Pagination : 47-61

ISBN : 2-9526131-1-7

\section{Référence électronique}

Laurent Dubreuil, «Alter, inter : académisme et postcolonial studies », Labyrinthe [En ligne], 24 | 2006

(2), mis en ligne le 24 juillet 2008, consulté le 08 mai 2019. URL : http://journals.openedition.org/ labyrinthe/1248; DOI : 10.4000/labyrinthe.1248 


\section{ALTER, INTER : ACADÉMISME ET POSTCOLONIAL STUDIES}

Laurent DubReuI

1d79@cornell.edu

Études ou théorie postcoloniales, ce vaste champ a été surtout constitué par des interventions émises sur les notoires campus américains, depuis une ou deux décennies. Ces enclaves dans le territoire des ÉtatsUnis ont permis le passage, la concentration, la circulation d'idées. Il est frappant de constater le poids de la diaspora indienne anglophone, mais le non-lieu de l'université américaine prime. Les francophones, plus ou moins intégrés à ce champ, comme Édouard Glissant, V. Y. Mudimbe ou Achille Mbembe, ont enseigné et travaillé on campus. La possibilité institutionnelle de créer des programmes transversaux par rapport aux départements (généralement disciplinaires), la nécessité de renouvellements périodiques dans les méthodes, l'importance accordée par les humanities à la theory (qui regroupe philosophie et épistémologie des sciences sociales), le rapport entre lutte des minorités et facultés depuis les années 1960, voilà autant de facteurs d'explication pour la cristallisation des études postcoloniales aux États-Unis. Les conditions de la mise au point d'un discours relèvent ainsi d'une situation nationale de l'enseignement et de la recherche. Le sens de la postcolonialité savante échappe en revanche à un unique déterminisme géographique. Dipesh Chakrabarty avance qu'il faut "provincialiser l'Europe», mais «dans un esprit de gratitude anticolonial ${ }^{1} »$. Le postcolonial exprime la possibilité d'une pensée qui fissure peut-être l'édifice rationnel et impérial, mais n'en cherche pas la démolition complète. L'affinité avec la position déconstructrice est patente, quoiqu'elle ne s'y résume pas. Le passage des frontières, fait colonial et geste de la pensée derridienne, est crucial

1. Voir Provincializing Europe. Postcolonial Thought and Historical Difference, Princeton, Princeton University Press, 2000 , p. 255. Ici et ailleurs, je traduis en français tous les textes écrits en langue étrangère. 
à ce titre. Il invite à remettre en cause les contingentements disciplinaires. Un pluriel de connaissances ne suffira pas.

Les postcolonial studies essaient donc de se doter d'un discours où l'objet et le sujet se changeraient mutuellement. Les auteurs entendent transformer la science comme le monde fut métamorphosé par le cours de l'histoire. Dans ce processus, ils comptent s'éprouver comme subjectivité dans l'interlocution textuelle. La théorie devient alors plus qu'un label chez quelqu'un comme Homi Bhabha. Elle est déjà une pratique, la démonstration par l'indigène que l'Occident n'a pas le privilège du concept. Cette désappropriation vise en retour la répartition même entre théorie et pratique. L'écriture de Bhabha, qui ramifie les phrases, glisse entre les synonymes, forge des néologismes, serait aussi une expression de l'hybridation de l'individu - et de l'Inde postcoloniale.

Le discours postcolonial est ainsi conçu comme l'instrument de savoir d'une parole adéquate. J'examine ici comment Gayatri Spivak et Homi Bhabha repensent les rapports entre disciplines à partir de l'émergence du subalterne. Bhabha et Spivak partagent une approche paradoxale. Ils témoignent d'un souci de l'autre, qui s'enracine dans leurs lectures philosophiques françaises. Spivak a traduit De la grammatologie de Derrida dès les années 1970, en lui ajoutant une présentation devenue presque aussi célèbre outre-Atlantique que le texte introduit. Elle termine sa Critique of Postcolonial Reason par un appendice sur l'œuvre de la déconstruction ${ }^{2}$. Bhabha cite abondamment Derrida et Lacan dans The Location of Culture, son livre le plus notable 3 . Chez eux, l'autre doit être préservé, et non pas réduit au même (occidental, masculin). Spivak reproche à Baudelaire de construire, dans «Le Cygne », un sujet marqué par la « cécité à l' autre femme » $(C P R, 152)$. Bhabha parle régulièrement d'une otherness, d'une autreté. Cette obsession de l'Autre, qui s'inscrit dans une lignée de la réflexion éthique depuis Lacan et Levinas, va alors s'articuler à la critique du devenir-autre tel que l'a pratiqué l'Occident colonial. Edward Said est la référence obligatoire sur ce terrain ${ }^{4}$. Spivak

2. Gayatri Chakravorty Spivak, A Critique of Postcolonial Reason. Toward a History of the Vanishing Present, Cambridge, Harvard University Press, 1999. Cité dans le texte par l'abréviation CPR, suivie du numéro de page.

3. Homi K. Bhabha, The Location of Culture, Londres, Routledge, 1994. Cité dans le texte par l'abréviation $L C$ suivie du numéro de page.

4. Voir Orientalism, New York, Pantheon Books, 1978 ; traduction française par Catherine Malamoud sous le titre L'Orientalisme. L'Orient créé par l'Occident, Paris, Seuil, 1980. Voir l'entretien avec Anne Berger et l'article d'Anthony Mangeon. 
et Bhabha font signe vers un autre qui ne serait pas le sous-homme oriental de l'impérialisme. Ils refusent donc l'altération hiérarchique, qui définit négativement. Le subalterne, le sous-autre, doit se refaire une étrangeté qui rompe avec l'unitarisme et la schize conceptuelle. Cette expérience, pour la théoricienne, pour le penseur, doit inclure un déplacement dans l'ordre du discours. Il s'agit de se rendre d'un point à l'autre, et de produire du sens entre les lieux d'énonciation. Alter va avec inter. Mais fort différemment de Spivak à Bhabha.

\section{L'alter-académisme}

Gayatri Spivak commence sa Critique of Postcolonial Reason par une remarque polémique, situant bien l'ambition réflexive de ses analyses. Les études postcoloniales, qu'elle a fortement contribué à assembler, sont d'après elle en danger de se banaliser. Si elles se cantonnent à la « représentation du colonisé ou au fait des colonies » $(C P R, 1)$, elles perpétuent le colonialisme, en niant la parole subalterne. De plus, institutionnellement, « les études des discours postcoloniaux/coloniaux sont en train de devenir un ghetto sous-disciplinaire substantiel » $(C P R, 1)$. Les chercheurs postcoloniaux pourraient tomber dans le piège, et accepter une place toute faite, qui ne touche en rien aux structures mêmes de l'Université. Spivak situe son ambition ailleurs. Au lieu de prendre le postcolonial pour en faire une semi-discipline, elle veut repartir du contingentement de la connaissance, puis échapper aux conditions méthodiques. Spivak ironique explique en préface : « Je ne suis pas assez érudite pour être interdisciplinaire, mais je peux briser les règles» $(C P R, \mathrm{XIII})$. Ce semblant d'humilité correspond à un projet particulier: habiter en étrangère chaque discours et s'y comporter autrement. L'informateur indigène de l'ethnologue (ou native informant, $C P R, 9$, passim) trouve son héritière dans un Je qui visite les disciplines pour, de l'intérieur, en montrer les points aveugles. Spivak partage sa Critique en quatre sections, intitulées Philosophie, Littérature, Histoire, Culture ${ }^{5}$. Elle pose des questions qu'elle pense inconvenantes. Elle assure qu'elle délivre des commentaires « fautifs » $(C P R, 9)$, quand elle scrute Hegel

5. Les deuxième et quatrième parties empruntent donc, en termes disciplinaires, à la critique littéraire et au groupe des cultural studies. 
du point de vue de sa connaissance approximative de l'Inde, par exemple. Elle opte pour le « sabotage » des disciplines (ibid.). Ainsi, elle se bâtit comme une présence perturbante, imprévue, au sein des institutions, et opère des rapprochements entre les oublis coloniaux de Kant ou de Baudelaire. Spivak reste une nostalgique de la littérature comparée, dont elle envisage l'agonie dans Death of a Discipline. Consciente de la prééminence de la littérature européenne dans l'histoire du comparatisme $^{6}$, elle se réjouit de l'ouverture par les area studies d'une complication de la donne. Mais elle voudrait préserver dans cette reconfiguration «le meilleur de la vieille littérature comparée: la capacité de lire les textes dans leur détail et leur langue originale ${ }^{7} »$. Et Spivak aimerait garder encore la comparaison. L'alignement des disciplines dans $A$ Critique of Postcolonial Reason permet une comparaison à deux registres. En passant d'un domaine à l'autre, le lecteur est conduit à entamer un travail herméneutique. Il lui revient de comparer le comparable (l'altération coloniale). L'agent de cette découverte est le Je-Spivak qui s'est formé dans le décentrement interne de chaque discipline; qui est produit par la comparaison avec les discours d'autorité (Marx et Brontë, les indianistes occidentaux et les scholars stars comme Frederic Jameson).

L'enjeu tient alors à la condition de formation d'un sujet postcolonial qui ne serait pas constitué par l'autre occidental, mais formulé au nom de l'altérité. Spivak cherche un autre autre, dont l'étrangeté est rendue évidente par les fautes qu'il commet contre les codes. Or, la difficulté tient dans ce soi-disant sabotage. Spivak brise-t-elle tant de règles qu'elle croit? Souligner la part coloniale de Rudyard Kipling, est-ce vraiment contraire à l'exercice de la critique littéraire? Non seulement l'approche dite idéologique est une vieille histoire, mais c'est aussi l'une des manières les plus courantes de ne pas lire dans le détail. Ailleurs, Spivak refuse deux interprétations divergentes de la phrase «Je pense à la négresse » dans « Le Cygne ». Selon elle, la négresse n'est ni un simple renvoi à Jeanne Duval, la maîtresse mulâtre de Baudelaire; ni un double du poète $(C P R, 155)$. La négresse est l'autre (indigène et féminin) qui paie le prix de la construction d'un je poétique blanc et masculin. En rejetant le commentaire référentiel comme la glose allégorique, Spivak n'est

6. Gayatri Chakravorty Spivak, Death of a Discipline, New York, Columbia University Press, 2003.

7. Id., p. 6. 
cependant pas la glorieuse saboteuse qu'elle se rêve. Elle prend en fait le parti d'une désignation littérale: la négresse est la négresse, exactement cela et rien d'autre, lisez comme c'est écrit. Les conclusions que tire Spivak en termes de domination n'ont rien de plus révolutionnaire; elles coordonnent l'examen du lyrisches Ich (typique de la germanistique) à une réévaluation politique du texte; tout cela a au fond un côté très RDA. Où est la subalterne indienne là-dedans?

Quand, plus loin, Spivak expose son désaccord avec «l'historien standard de l'Inde » $(C P R, 222)$, elle prend soin de dire qu'elle ne connaît que le standard. Elle précise: «Il doit être absolument clair que je ne suis pas une historienne » et qu'elle est dépourvue d'un « jugement mature sur le plan disciplinaire » $(C P R, 222)$. Cette attitude me trouble infiniment, je l'avoue. L'immaturité n'est-il pas un thème central du colonialisme (et de toute chefferie s'exerçant sur un peuple à qui l'on retire le pouvoir)? Les Nègres pas assez mûrs pour être libres, les Orientaux pas assez mûrs pour se gouverner eux-mêmes. Le sabotage, je veux bien, mais pourquoi revendiquer l'immaturité? Pourquoi ne pas faire imploser l'histoire en se faisant historienne? Pratiquer une discipline sans détenir de diplôme d'attestation et hors des facultés autorisées me semble nettement plus déstabilisant pour le monde universitaire que la reconnaissance des autorités en préalable à leur critique. Enfin, consentir au seul standard et ignorer les positions marginales et minoritaires est une façon bien pratique de réserver la critique à l'expression subalterne. Autrement dit, Spivak, à dessein, contresigne les grandes proclamations unitaristes occidentales, elle rejoint la tradition majoritaire pour qui les autres n'existent pas - afin de s'arroger une stratégie contestataire. Les disciplines, dans leur histoire, ne tiennent que par la controverse; sinon, elles s'effondrent en doctrines. Mais assurément, à chaque instant, un écrasant consensus fait comme s'il détenait seul la parole. L'omission du mouvement adverse dans la vie des disciplines consent donc à la standardisation finale du savoir, par-delà les reproches adressés aux historiens standards.

L'entreprise de sabotage ne prend donc de sens que par rapport à une chose : Gayatri Spivak, femme, Indienne, professeur, dérange l'ordre de la connaissance par le seul fait qu'elle s'exprime. Car dans le discours, les dérèglements annoncés se révèlent au contraire la reconnaissance tacite de l'exercice disciplinaire (acceptation du contrôle normatif de la connaissance, recours en masse à des catégories opératoires éprouvées, 
réappropriation de la critique interne). Le je subalterne est préformé par l'histoire que la personne de l'auteur pense porter, et cela suffit à déterminer l'ampleur de la critique. Là seul se situe l'erroné, le fautif, qui, dans le livre de Spivak, n'est pas effectivement accompli.

Depuis une posture individuelle, irréductible et préalable au langage, on se contentera de comparer les différents discours de l'autre, de faire entendre une voix talentueuse, assez insolente pour capter l'attention et assez respectueuse pour faire florès dans l'Université telle qu'elle est. Aucune bombe n'éclatera, aucun train ne déraillera, les saboteurs ne détruiront rien. L'Academia au sens anglo-saxon n'a plus qu'à faire place à la postcolonial critique. Elle est sommée de ne pas inférioriser, de ne pas créer de ghettos sous-disciplinaires. Mais, miroir de l'univers, l'Université acceptera désormais la différence. Spivak a récemment assuré que «l'avenir des humanities est un monde fragmenté ${ }^{8} »$. Ce futur entérine les répartitions disciplinaires et les secoue légèrement par l'introduction (de principe, mais pas de fait) d'une altérité constituée. La parcellisation du monde est une réponse à la globalisation économique et politique, vue comme néocolonialisme. Un autre monde est possible, dit l'alter-mondialisme. À la suite, Spivak prophétise un alter-académisme. Une pluridisciplinarité éventuellement imparfaite aura pour mission de donner lieu à un subalterne prédéfini : les facultés se retrouveront de temps à autre sous l'égide d'un comparatisme ressourcé. Le discours résultant se coulera alors dans une prose académique énoncée au profit des autres, de plus en plus vaguement saisis. Les phrases de convention peuvent s'empiler, comme «il est évident que l'intérêt personnel conduit la mondialisation ${ }^{9} \gg$.

Sous ses deux aspects, l'alter-académisme, dans le contexte américain qui lui donne forme, ne brise aujourd'hui aucune règle. Il est logique que Gayatri Spivak occupe une fonction de premier plan au sein des humanities à Columbia University. Bien sûr, transposée dans un milieu comme l'université française, la critique postcoloniale semblerait dévastatrice, ou radicalement révolutionnaire. Elle remettrait en cause une politique nationale des carrières et les intérêts réciproques des micropouvoirs spécialisés. Pourtant, même dans l'amphithéâtre Richelieu en

8. Dans « Guest Column : Roundtable on the Future of the Humanities in a Fragmented World », PMLA. Publications of the Modern Language Association of America, mai 2005, vol. CXX, n 3, p. 720. 9. Ibid. 
Sorbonne, le geste de Spivak demeurerait inoffensif pour l'architecture intellectuelle des disciplines. Les erreurs de perspective chez Hegel ou Kant viennent de leur position individuelle dans l'histoire, et pas de la philosophie. Cette dernière discipline sera frustrée du désintéressement qu'elle affiche, mais il y a longtemps qu'elle s'habitua à sa relativité, sans ciller beaucoup. La critique subalterne, malgré qu'elle en ait, ne transgresse aucune règle disciplinaire. Elle liste des griefs pour assurer son existence par sa loquacité ; et elle adresse aux autres mondes les éléments d'un perfectionnement de l'appareil épistémique.

Les scholars postcoloniaux de Spivak se situent dans l'intersection de plusieurs traditions, mais, plus particulièrement responsables d'un fragment de l'espace, ils visitent les autres en gardant leur étrangeté (non questionnée). Alter va avec inter, dans l'interruption de la totalité universaliste, et les intervalles ouverts entre les différentes parties du monde. Cette postcolonialité-là entérine les partages (du savoir, de l'univers) acquis avec l'expansion européenne puis la chute des empires. L'autre autre demeure un projet du sujet, tel que les traditions conceptuelles majoritaires d'Occident l'ont formé. Chez Spivak, la réforme postcoloniale des discours épistémiques inclut donc un usage décalé des disciplines. Ce décentrement (ni faute, ni sabotage) permet de prolonger, dans l'institution du savoir, l'étrangeté empirique qu'éprouvent les (dé)colonisés dans un territoire que perturbe la présence impériale. Comment s'édifie cette expérience, comment un propre se constitue, comment un sujet serait à même de se former avant la parole - Spivak ne nous en dit rien. Son silence explique le registre double de son usage disciplinaire (mise en garde contre la dangerosité et effective innocuité).

\section{L'intervalle subalterne}

Ces problèmes, en revanche, se trouvent au cœur de la démarche de Homi Bhabha. L'autreté est recherchée de telle sorte que la reconstitution d'un sujet plénier (fût-il autre) soit impossible. Dans The Location of Culture, la maxime "Ni l'Un [...] ni l'Autre [...] mais quelque chose d'autre au-delà » (cf. LC, 28) repousse le simple renversement identitaire comme l'altération et pose la nécessité d'un pas en supplément. Le passage par les frontières conceptuelles implique une identité fracturée qui ne se contente pas de l'empirique étrangeté prédiscursive. L'au-delà 
postcolonial, qui se nomme l'hybride, « conteste les termes et les territoires de » l'un et l'autre (ibid.). Cette position, Bhabha la veut théorique et pratique. Il glose ainsi l'Un et l'Autre par des références aux luttes politiques (respectivement « classe ouvrière unitaire » et « les politiques des sexes »). «L'engagement pour la théorie » qui donne le titre du premier chapitre reprend à son compte la volonté marxienne d'un changement du monde par la philosophie. La célèbre citation de Marx figure d'ailleurs en bonne place dans Peau noire, masques blancs de Frantz Fanon ${ }^{10}$, l'un des penseurs cruciaux pour la réflexion de Bhabha. Penser l'hybridation aiderait à reconfigurer les rapports politiques entre les mondes, les peuples et les sujets. L'ouverture à l'excès dans la dynamique identitaire fait reconnaître une "différence culturelle [comme] le processus de l'énonciation de la culture » $(L C, 34)$. Bhabha sépare ici la différence culturelle de la cultural diversity, fondée sur la circonscription de « contenus et de coutumes pré-donnés » (ibid.). Au contraire, l'identité subjective, la culture sont envisagées dans le mouvement d'une parole qui change le locuteur. L'enjeu politique se traduit alors par une phrase : « La reconnaissance théorique de l'espace éclaté de l'énonciation peut ouvrir la voie à la conceptualisation d'une culture internationale, fondée non pas sur l'exotisme multiculturel de la diversité des cultures, mais sur l'inscription de l'hybridité de la culture » $(L C, 38)$. La bataille pour « la différence des autres cultures » est reléguée au rang des « stratégies théoriques qui sont nécessaires pour combattre "l'ethnocentrisme" » $(L C, 70)$; mais, sous son avatar relativiste ou indigéniste, elle loupe « l'excès de signification » qu'est « l'autreté » (ibid.). Autreté plutôt qu'altérité. Le terme de Bhabha, otherness, est un peu moins rare que le mot français que je lui associe ; il n'en reste pas moins guère usité. Je choisis le néologisme afin de souligner la recherche lexicale de Bhabha et sa volontaire mise à distance de alterity (altérité) ou strangeness (étrangeté). Il est à noter qu'en 1936, Antonio Machado, forgeait le néologisme d'otredad (autreté) afin de désigner une hétérogénéité de l'être, une ouverture du sujet à l'Autre ${ }^{11}$. Le concept sera repris par Octavio Paz : comme «l'autreté est dans l'homme même ${ }^{12}$ », par la "révélation poétique », Je devient capable d'être un autre. Pas complètement inconnue

10. Paris, Seuil, 1971, p. 13.

11. Manuel et Antonio Machado, Obras Completas, Madrid, Editorial Plenitud, vol. II, 1967, p. 1120. 12. Voir El Arco y la Lira, Mexico, Fondo de Cultura Economica, 1956, p. 173. 
dans une langue romane proche, l'autreté entretient donc bien des rapports avec la fracture du même par l'otherness, aux confluents de la parole et de l'éthique.

Que les propos théoriques de Bhabha se transcrivent aisément en mots d'ordre politiques resterait à voir. On peut du moins accorder à Bhabha la recherche du politique dans le faufilage de son texte. L'autre (post)colonial se fait international : l'hybride se reconstruit alter dans un inter-espace. L'analyse de la différence culturelle doit ainsi recourir à une «transformation interdisciplinaire et discursive » $(L C, 163)$. L'interdisciplinarité n'est point confondue avec un pluriel, « ce n'est jamais simplement une harmonieuse addition de contenus ou de contextes qui augmente la positivité d'une présence disciplinaire ou symbolique, donnée d'avance » (ibid.). Elle repose plutôt sur les intervalles entre savoirs, elle crée de nouvelles fêlures qui désorganisent l'architecture. On n'imagine pas The Location of Culture se séparer en plusieurs parties, nommées « Philosophie » ou « Histoire ». Au contraire, Bhabha reconnecte de manière originale Lacan et Walcott, Fanon et Lévi-Strauss, etc.

Cette pratique de l'excès disciplinaire dans la forge d'un nouveau sens se présente comme une obligation pour l'expression épistémique de l'hybridation coloniale. Le lien entre interdisciplinarité et sujet postcolonial est si intense qu'il demande un bref exposé de l'argumentation de Bhabha sur l'ouverture de l'identité. «The Other Question » (question de l'Autre et questionnement autre) est posée par la colonie. Les indigènes se retrouvent dans une situation complexe où le pouvoir central les agrège à un corps national, dont ils sont parallèlement exclus. Les colonisés se rattachent à l'empire, alors que leur différence (statutaire, raciale) est en permanence marquée. Bhabha trouve dans ce doublethink (pensée double, $L C$, 111) colonialiste les germes d'une modification imprévue. Par la loi et le simple quotidien, les indigènes face aux colons sont " presque le même mais pas tout à fait » ( $L C, 89$ et passim), ou encore « moins qu'un et double » (LC, 97 et passim). L'ambivalence colonialiste produit un écartèlement, qui a aussitôt un grand retentissement. Car, pour Bhabha, le double registre réalise l'impasse du discours colonial, et son incapacité puriste à penser la réalité qu'il met en place. Je ne jurerais pas que l'événement de la contradiction soit si périlleux pour le colonisateur ; mais j'y reviens bientôt et retourne pour l'instant à l'argument du livre. 
L'hybride fait fond en cette sommation impossible (moins que l'un et double). Il y puise la force d'une logique autre, qu'il parle comme nativement. Il s'ensuit un sujet du discours qui réévalue les oppositions binaires, les hiérarchies, les puretés impures. Le fait est toujours processuel. Le postcolonial ne procède pas d'une réunion ou d'une synthèse d'éléments préexistants. Il se forme par un discours dont l'effectivité ruine les oppositions. Il imite le colonial, comme on le lui demande. Mais en imitant, il contrevient également au désir solitaire du colonisateur : la ressemblance, parfaite et pourtant interdite, donne lieu à une autreté qui n'est pas l'altération, qui doit être sans cesse continuée dans l'avenir d'une identité. Avec la mimicry, Bhabha traverse les textes de Lacan sur le miroir et l'identité. Il essaie de poser une imitation dérangée, dérangeante, qui ne soit pas le mimétisme colonial du bon Noir faisant comme le Blanc. La légende du psittacisme est rendue à la virulence d'une imitation tirant sa puissance des règles d'imperfections qui l'enserrent.

\section{Le tiers hybride}

L'interdisciplinarité dans The Location of Culture entretient la performative transformation du sujet indigène Bhabha. Faisant presque la même chose que l'Academia occidentale, il ne l'accomplit pas complètement. Sa voix ne monte pas depuis une région autre dans le continent des disciplines ; elle imite et modifie. Ainsi Bhabha associe-t-il l'excès des savoirs à la démonstration de l'hybridation.

Les difficultés théoriques de la catégorie deviennent par ce biais les limites épistémologiques de l'interdisciplinarité. Il nous faut scruter la logique hybride dans son fonctionnement. Une première assertion nous arrêtera dans ce retour à la création de l'hybride: « 1 n'est pas autocontradictoire mais, dans le processus de sa discussion, il réalise de manière signifiante les problèmes de jugement et d'identification qui informent l'espace politique de son énonciation » $(L C, 29)$. On aurait pu trouver la description d'un colonisé qui ne soit ni l'un ni l'autre, mais l'un et l'autre. Non. Pas de contradiction; elle est occidentale, et contreproductive pour elle-même, et involontairement propice à son bouleversement. Répétons : l'hybride n' est pas contradictoire. Telle insistance fait un curieux couple avec l'incessante répétition du terme d'identité. Je sais qu'en Amérique particulièrement, les locuteurs comprennent l'identité 
d'abord comme une relation à l'autre. (Si des étudiants des États-Unis vous passent entre les mains, faites l'essai à votre tour, demandez-leur de définir le substantif, et vous verrez.) Je sais aussi que Bhabha parle d'ouverture du sujet. Néanmoins, une identité non contradictoire, voilà qui ne me paraît pas si loin des principes qu'Aristote écrivait au début de sa Métaphysique.

Un peu plus loin. L'hybride, intervalle entre l'un et l'autre, n'est pas un « troisième terme résolvant la tension » entre les opposés $(L C, 113)$. Tiers terme, disqualifié, vaut donc pour synthèse, pour le dépassement hégélien. Mais je crois très précisément que l'hybride est du côté du tiers terme - tel que l'évoque Platon dans le Timée. Triton allo genos : troisième autre genre ( $c f$. Timée, 48e-49a). Dans ce célèbre passage du dialogue, Socrate, qui discute de l'univers, s'intéresse à la formation de la matière. Lui et son interlocuteur avaient auparavant distingué le modèle intelligible (paradeigma) et la forme sensible qui le reproduit par imitation (mimêma). Il convient, estime Socrate, d'ajouter un troisième genre, autre, qui rende possible l'impression de la matière. Ce qui s'appelle khôra, à la fois étrange région et matrice. Le mot de khôra a connu une vogue dans les dernières décennies du $\mathrm{XX}^{\mathrm{e}}$ siècle, à cause des commentaires qu'en firent Jacques Derrida et Julia Kristeva. Je souligne cette mode non pour me réclamer de l'une de ces interprétations (ou de celles qu'elles bercèrent); plus pour indiquer, dans l'histoire des idées, un autre lieu de passage intellectuel.

Platon, donc, à sa théorie de l'imitation, annexe un tiers genre, très « difficile » à concevoir (Timée, 49a), qui ne soit ni l'un (noétique et supérieur), ni l'autre (copie inférieure, subalterne); cf. Timée, 52a-52b. Le triton allo genos n'est nullement synthétique, il est produit par le raisonnement de Socrate comme une nécessité contrevenant à la dualité dans le processus mimétique. La khôra est un lieu étonnant entre l'un et l'autre. Ou doit-on dire, avec Bhabha, «le topos de l'énonciation» hybride $(L C, 162)$ ? La sollicitation du grec (topos) dupliquerait le jeu de mots (topos, lieu et lieu commun) de khôra (endroit et matrice).

Voici que l'hybride de Homi Bhabha se rapproche davantage du Timée. Socrate affirme, au bout de sa discussion sur la matière, que le troisième autre genre se laisse saisir, à grand mal, par un nouveau mode de pensée. Il lui faut un mixte entre «l'intellection » (noèsis) et « l'opinion alliée à la sensation » (doxèi met'aisthèseôs) ; Timée, 52a. Alias : il faut passer par logismôi tini nothôi, « une sorte de raisonnement bâtard », 
dont la philosophie n'a pas grand-chose à dire, tant il passe ses bornes (Timée, 52b). L'expression grecque doit retenir. Logismos dit raisonnement, et dérive de logos, qui renvoie aussi à la parole, au langage. Quant à nothos, l'adjectif désigne l'indistinct, le douteux, le contrefait. Et, avant cela, le bâtard, oui, l'enfant qu'un maître donne à son esclave (dans l'Iliade). Dans l'âge classique, est nothos le rejeton de l'Athénien et de l'étrangère. Plutarque renvoie à cette catégorie politique dans sa Vie de Thémistocle (§I) : Thémistocle était nothos par sa mère étrangère. Jacques Amyot, en 1559, traduit par mestifis (métisse), à une époque où le terme se charge de son sens colonial dans les langues romanes. Pour le tiers genre, il faut s'outiller d'un raisonnement louche comme le métissage des peuples et des mondes, d'une pensée hybride - comme l'antique traduction Budé choisissait de le dire ${ }^{14}$.

Oh bien sûr, Platon ne compte pas fonder toute sa philosophie sur l'hybride. Puis il suspecte l'intelligibilité de ce tiers non synthétique. Enfin, sa khôra est matricielle. Il n'empêche, j'ai l'impression que l'hybride de Socrate et celui de Bhabha ont un air de famille. Est-ce gênant? Éliminons d'un coup l'hypothèse (néo)coloniale - Bhabha ne faisant que répéter, au mieux acclimater Platon. Reste l'explication que fournirait la mimicry postcoloniale. Bhabha, sujet hybride, se livrerait à une imitation et différence qui changeraient le point d'articulation du discours philosophique. Mais en quoi disposons-nous d'un discours qui « transforme les conditions d'énonciation au niveau du signe » $(L C, 247)$ ? Je mets à part l'assignation biographique du locuteur indigène, elle ne saurait suffire dès lors qu'il s'agit du processus de la parole et que l'on réfute «l'identité pré-donnée» $(L C, 45)$.

Bhabha reconduit Platon quand il tient l'hybride pour non contradictoire afin de tenter sur lui un discours. Quand il propose une logique à même de dire le troisième autre genre. Quand il critique la dégradation mimétique au profit d'un lieu d'imitation différent. Quand il se dote d'une pensée hybride, métisse, pour désigner ce qui échappe à la bipolarité. Bhabha modifie l'argumentation de Platon, voire la métamorphose, d'accord. Mais chaque fois que Platon est retourné de fond en comble, mis sens dessus dessous, ouvert et recousu autrement, l'opéra-

13. Cf. Plutarque, Les Vies des hommes illustres, traduction français Jacques Amyot, Paris, Gallimard, 1951, vol. I, p. 246.

14. Intercesseur possible, Jacques Derrida rappelle une partie de ce champ sémantique dans Khôra, Paris, Galilée, 1993, p. 17. 
tion s'appelle néoplatonisme. Autre Marsile Ficin, autre Plotin, Homi Bhabha trouve en Platon de quoi le refaire pour son temps et sa Cité. Je dis cela avec une grande admiration pour le texte habilement retors de Bhabha. Juste, je m'interroge sur cette nécessité postcoloniale (historique et géographique) qui aurait créé l'hybridation. À moins que Platon ne fasse partie de ces œuvres européennes xénophiles, dont il est parlé dans le premier chapitre (avec Montesquieu, Barthes, Lyotard...). Pourquoi pas ? mais, à ce compte, qui resterait dans le camp du « signe logocentrique occidental » $(L C, 31)$ ? Personne et tout le monde, car l'Occident n'existe pas plus, ni mieux, que l'Orient.

En somme, soit l'on accorde à Bhabha le privilège d'une hybridation malgré tout par le simple fait qu'Indien postcolonial, il modifie la parole. En ce cas, la démonstration deviendrait exclusive - aboutissement peu crédible puisque les dernières lignes de The Location of Culture font résonner quatre fois human. Soit la transformation de l'énonciation ne correspond pas à l'analyse donnée, car, en fait, la parole, même en Europe, n'a jamais été uniment le logos rationnel ; et l'autre n'a pas été toujours muet, ou trouvé aux îles. Soit la reconduction différentielle est postcoloniale, car émise dans l'interzone de Naipaul, Fanon, Derrida ou Foucault; mais elle l'est potentiellement, pas foncièrement. L'hybride désigne une expérience sortie de l'empire, et une autre différente, et ainsi de suite. Alors, le postcolonial (l'imitation manquée, le déni d'humanité ou de citoyenneté) perd sa qualité principielle, il se mue en circonstance.

Je n'exclus pas que les trois résolutions que je viens de donner puissent s'articuler. Pour moi, il y a une pente académique de Homi Bhabha, Académie nommant le lieu d'énonciation de tous les platonismes. L'inclinaison n'invalide pas le raisonnement, mais elle le sépare de l'unicausale colonie. Par suite, la détermination interdisciplinaire du discours aura grand mal à se réclamer de l'intervalle ou de l'interstice produits par les sujets hybrides. L'inter se raccorde à une logique académique (platonique) du tiers terme, qui rationalise le non-rationnel tout en mêlant les types de connaissances. Pareil projet, dans l'Academia anglosaxonne, reste à la fois possible et hors norme. Il ne me semble toutefois pas produit par l'unique hybridation postcoloniale.

La recherche de Bhabha diverge entre son dit et son dire. L'interdisciplinarité n'a rien de fortuit, mais son ambition doit se distinguer du concept d'hybridation qu'elle explique. Elle semble d'abord motivée par la réalité institutionnelle des studies. Les formes nouvelles dans l'uni- 
versité américaine et anglo-saxonne passent en général par des conglomérats interdépartementaux. Bhabha outrepasse alors la logique du multiple, où Spivak décidait de demeurer. L'inter n'est cependant pas commandé par l'hybride, ni secrété par une nécessité interne à l'énonciation postcoloniale. Si l'interdisciplinarité est réappropriée par Bhabha, il n'y a pas de nature coloniale contraignante pour la création d'un inbetween - la reconduction néoplatonicienne du tiers le montrait. Par complément, la croisée des disciplines est une exigence de la pensée qui correspond à un moment dans la production historique des discours. La postcolonialité est saisie au sein de ce phénomène d'époque, elle n'en est pas le déterminant. Sans doute Bhabha sacrifie-t-il ici à une tentation courante. Il cherche à refonder une méthode en fonction du problème qu'il doit traiter. Il s'attache à établir la cohérence complète de sa théorie pratique.

Et si nous renoncions à ce type de cohésion ? Ce au profit d'une adéquate inadéquation, qui ne manquera pas de se faire jour. Bhabha avec la théorie et Spivak avec la critique désignent bien l'espace d'intervention intellectuel où la diction épistémique de la colonie se passe. Nous pouvons revendiquer un discours théorique critique. Je n'entends pas refaire la doctrine de l'École de Francfort. Je rappelle juste que l'Institut für Sozialforschung fut le lieu d'un vrai échange entre disciplines. La place accordée à la littérature et la philosophie me retient encore. La kritische Theorie s'attaquait en particulier à la contrainte sociale et au pouvoir. Max Horkheimer la situait ainsi à l'encontre du « conformisme de la pensée ${ }^{15} »$. Ces question conservent leur pleine valeur aujourd'hui, indépendamment des doctrines (au demeurant fort dissemblables) qui furent émises par les Francfortois sur plusieurs décennies.

Il me parait important que le théorique critique passe encore par la critique de la théorie. Cela inclut un questionnement des institutions de la connaissance et des méthodes ou discours. Je prêche pour une excessivité critique, qui est le temps de notre pensée. Sur la colonie - mais pas seulement -, l'objectif est de passer les disciplines au crible, et d'en outrepasser les protocoles définitoires. L'indiscipline est un terme dont nous jouons depuis un moment dans Labyrinthe ; il faillit servir de soustitre à la revue dès 2001, figure dans l'argument à partir du numéro 14

15. Max Horkheimer, Traditionnelle und kritische Theorie. Vier Aufsätze, Francfort, Fischer, 1968, p. 56 [article de 1937]. 
(au début de 2003), et apparut en première page de notre dossier dédié à Jacques Rancière $\left(n^{\circ} 17,2004\right)$. Labyrinthe n'étant pas une publication à manifeste, les membres de la rédaction ne sont pas tout à fait d'accord sur l'indiscipline, et ne cherchent d'ailleurs pas à penser pareillement. Je reprends dans l'indiscipline la marque d'une négativité épistémique qui résulte de la mise en forme méthodique. Pas de négation a priori. L'apprentissage des disciplines les rend caduques en un certain point. Là, il s'agirait d'user des multiples défauts pour nous projeter dans une énonciation hétérodoxe. Il est inepte de prétendre dédisciplinariser si l'on veut dire rejeter avant toutes choses les coercitions méthodiques. La parole native, l'originalité dogmatique sont des constructions historiques, au même titre que la sociologie ou la philologie; et pas forcément plus productives dans l'épreuve de leurs limitations. Mieux vaut établir, en acte, l'incapacité disciplinaire (et, a fortiori, l'impuissance de la spécialisation) afin d'en tirer la force d'une réforme. Ainsi se dote-t-on d'une théorie de sa propre critique. Comprenons de quoi nous nous séparons, et pourquoi. 